\title{
Prolonged diagnostic delay in acromegaly is associated with increased morbidity and mortality
}

\section{Daniela Esposito ${ }^{1,2}$, Oskar Ragnarsson 1,2, Gudmundur Johannsson ${ }^{1,2}$ and Daniel S Olsson ${ }^{1,2}$}

${ }^{1}$ Department of Internal Medicine and Clinical Nutrition, Institute of Medicine, Sahlgrenska Academy, University of Gothenburg, Gothenburg, Sweden and 2Department of Endocrinology at Sahlgrenska University Hospital, Gothenburg, Sweden
Correspondence should be addressed to $D$ Esposito

Email

daniela.esposito@gu.se

\section{Abstract}

Context: Clinical features of acromegaly develop insidiously. Its diagnosis may therefore be delayed.

Objective: Our aim was to study diagnostic delay and its impact on morbidity and mortality in a nationwide cohort of patients with acromegaly.

Design: Adult patients diagnosed with acromegaly between 2001 and 2013 were identified in the Swedish National Patient Registry. Diagnostic codes for predefined comorbidities associated with acromegaly were recorded between 1987 and 2013. Diagnostic delay was calculated as the time between the first registered comorbidity and the diagnosis of acromegaly.

Results: A total of 603 patients (280 men, 323 women) with acromegaly were included. Mean (s.D.) diagnostic delay was 5.5 (6.2) years (median (minimum, maximum) 3.3 (0.0-25.9)) Diagnostic delay was $1-<5$ years in $23 \%$ patients; $5-<10$ years in $17 \%$; and $\geq 10$ years in $24 \%$. No delay was recorded in $36 \%$ of patients. Overall, mean (s.D.) number of comorbidities was $4.1(2.5)$ and was higher in patients with longer diagnostic delay $(P<0.0001)$. Overall, observed number of deaths was 61 (expected 42.2), resulting in a standardized mortality ratio (SMR) of 1.45 (95\% Cl: 1.11-1.86). Increased mortality was only found in patients with the longest diagnostic delay (1.76, 95\% Cl: 1.12-2.65). In the other groups, no statistically significant increase in mortality was recorded, with the numerically lowest SMR observed in patients without diagnostic delay (1.18; 95\% Cl: 0.68-1.92).

Conclusions: The diagnosis of acromegaly is delayed in most patients. Prolonged diagnostic delay is associated with increased morbidity and mortality.

\section{Introduction}

Acromegaly is a rare disease, most commonly caused by excess growth hormone $(\mathrm{GH})$ secretion from a pituitary adenoma (1). Excess GH and consequently increased insulin like growth factor 1 (IGF-1) result in typical acromegalic features, including acral enlargement, prognathism, growth of skin and connective tissue, visceromegaly, and so forth $(1,2,3)$.
The clinical features of acromegaly develop insidiously over time and its diagnosis is often delayed $(1,4)$. At diagnosis, most patients present with one or multiple comorbidities such as metabolic, cardiovascular, and respiratory disorders, as well as malignancies, that may lead to excess mortality $(3,4,5,6)$. Biochemical remission has been shown to normalize mortality rate in patients 
with acromegaly (7). Nevertheless, biochemical control may only mitigate rather than reverse the associated complications.

Diagnostic delay in acromegaly seems to vary greatly across studies. In the 1960s, a case series, aimed at describing the clinical features of patients with acromegaly, reported a diagnostic delay of 10-20 years (8). In the 1980s, a larger case series described a mean diagnostic delay of 9 years (9). Despite improvements in the diagnostic procedures for acromegaly (e.g., the availability of serum IGF-1 as a simple diagnostic test), the diagnostic delay and the age at diagnosis do not seem to have improved over recent decades $(10,11,12,13)$, with recent national multicentre studies showing a delay that ranges between 5 and 14 years $(11,12,13)$. None of these studies was however specifically designed to analyse diagnostic delay. Moreover, the diagnostic delay was estimated through medical interviews, or questionnaires, for example, techniques that are to some extent inaccurate because of susceptibility for recall bias.

Diagnostic delay in acromegaly may be associated with an increased risk of comorbidities, presumably leading to excess mortality. To our knowledge, no data are available on the impact of diagnostic delay on longterm outcome. The aim of this study was to investigate the delay in the diagnosis of acromegaly and its impact on morbidity and mortality in a nationwide unselected cohort of patients with acromegaly in Sweden.

\section{Subjects and methods}

\section{Study design}

Data were retrieved via linkage of national healthcare registries, which cover the entire Swedish population. Patients with acromegaly were identified in the Swedish National Patient Registry (Patient Registry), which achieved national coverage in 1987. The Patient Registry contains information on every patient visit or admission within the national hospital system, including data on surgical treatment and radiation therapy. Causes of death were collected from the Swedish Cause of Death Registry (Cause of Death Registry), which was established in 1952. Information on medical therapy was retrieved from the Prescribed Drug Registry (Drug Registry), which includes data on all prescribed medical treatments purchased in pharmacies in Sweden since 1 July 2005. Via the personal identification number, which is unique for every Swedish resident, it is possible to follow an individual patient through life and collect exhaustive healthcare data from the national registries. The National Board of Health and Welfare guarantees a high quality of the data in the national registries $(14,15)$.

The study was approved by the Regional Ethical Review Board in Gothenburg, Sweden and by the National Board of Health and Welfare, Sweden.

\section{Study population}

The study population included all adult patients $(>18$ years of age at diagnosis) with an acromegaly diagnosis due to a pituitary tumour established in Sweden between 1 January 2001 and 31 December 2013. To ensure high accuracy in the patient selection process, a combination of search criteria was used: the International Classification of Diseases (ICD-10) codes for acromegaly (E22.0) in combination with neoplasms of pituitary gland (D35.2 or D44.3). Only patients with both diagnoses were included in the study. The pituitary tumour diagnosis should have occurred either up to 5 years before the first acromegaly diagnosis or any time after the reported diagnosis of acromegaly (E22.0). Individual diagnosis was set after a clinical decision-making process for each patient.

Based on a previous study from our group (3) and current knowledge $(1,2)$, diagnostic codes for predefined comorbidities associated with acromegaly were retrieved from the Patient Registry (Supplementary Table 1, see section on supplementary materials given at the end of this article). Each patient was followed for 14 years before the diagnosis of acromegaly, and, after the diagnosis, until death or the end of the study (31 December 2013), that is, the entire cohort of patients had equally long time to develop any of the predefined acromegalic comorbidities (time at risk) (Table 1).

The date of diagnosis was defined as the first specialized healthcare visit or admission with acromegaly as the main or secondary diagnosis. The date of onset of the first comorbidity was defined as the first registration of any predefined comorbidity (Supplementary Table 1). The delay to diagnosis was calculated as the time between the date of the first registered comorbidity and the date of acromegaly diagnosis. Comorbidities were also clustered into disease categories (Supplementary Table 1) and the diagnostic delay was calculated for each category. In addition, a sub-analysis of diagnostic delay was preformed based on a stricter selection of more specific acromegalyassociated complications (Supplementary Table 2).

Patients were categorized based on the delay to diagnosis (Fig. 1). Since it is practically impossible to have a diagnostic delay equal to zero, patients with the first 
Table 1 Characteristics of patients with acromegaly in Sweden diagnosed between 2001 and 2013.

\begin{tabular}{l} 
Characteristics \\
\hline Age at diagnosis, mean (S.D,) \\
Age at first comorbidity, mean (S.D.) \\
Time from comorbidity onset to diagnosis, years \\
$\quad$ Mean (s.D.) \\
Median (min, max) \\
Follow-up time, mean (s.D.), years* \\
Time at risk, median (min, max), years ${ }^{\ddagger}$ \\
No. of comorbidities up to diagnosis, mean (S.D.) \\
No. of comorbidities at any time, mean (S.D.) \\
Patients with hypopituitarism, $n$ (\%) \\
Treatment with surgery, $n(\%)$ \\
Treatment with radiotherapy, $n$ (\%)
\end{tabular}

\begin{tabular}{c}
\hline Total $(n=603)$ \\
\hline $51.8(15.3)$ \\
$46.8(15.1)$ \\
$5.5(6.2)$ \\
$3.3(0.0,25.9)$ \\
$12.2(6.6)$ \\
$27.0(14.2,27.0)$ \\
$1.9(1.8)$ \\
$4.1(2.5)$ \\
$193(32.0)$ \\
$418(69.3)$ \\
$74(12.3)$ \\
\hline
\end{tabular}

\begin{tabular}{l}
\hline \multicolumn{1}{c}{ Men $(n=280)$} \\
\hline $52.1(14.1)$ \\
$47.8(13.9)$
\end{tabular}

\begin{tabular}{lll}
\hline Women $(n=323)$ & & P value \\
\cline { 1 - 1 } $51.6(16.2)$ & & 0.73 \\
$46.0(15.9)$ & & 0.15
\end{tabular}

*Follow-up time, time from the first registered acromegaly-related complication to death or end of the study (31 December 2013); ${ }^{*}$ Time at risk, Each patient was followed for 14 years before the diagnosis of acromegaly, and, after the diagnosis, until death or the end of the study (31 December 2013).

comorbidity occurring $<1$ year before acromegaly diagnosis were categorized in the group Without Diagnostic Delay. These patients were grouped together with patients without comorbidities and those with comorbidities registered any time after acromegaly diagnosis (Fig. 1). Group Diagnostic Delay $1-<5$ included patients with comorbidity occurring 1 to $<5$ years before the diagnosis of acromegaly; group Diagnostic Delay $5-<10$ included those with comorbidity occurring 5 to $<10$ years before the diagnosis; and group
Diagnostic Delay $\geq 10$ those with comorbidity occurring $\geq 10$ years before the diagnosis (Fig. 1). To study whether diagnostic delay had an impact on the management and treatment of acromegaly, information on surgical procedures and radiation therapy was collected from the Patient Registry for each group. Data on medical treatment were collected for a subgroup of patients who had received acromegaly diagnosis after 1 July 2005, when the Drug Registry achieved nationwide coverage in Sweden. The

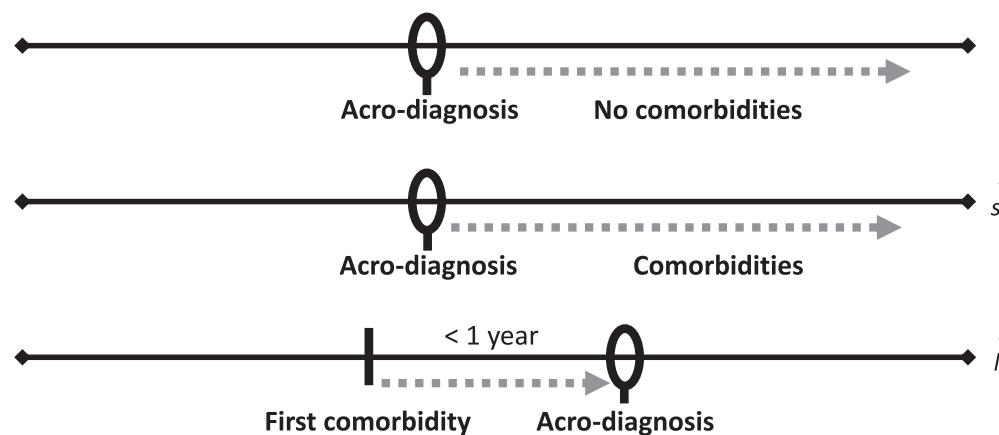

Patients without comorbidities $(n=24)$

Patients with onset of comorbidity same date as acromegaly diagnosis or any time after $(n=128)$

Patients with onset of comorbidity

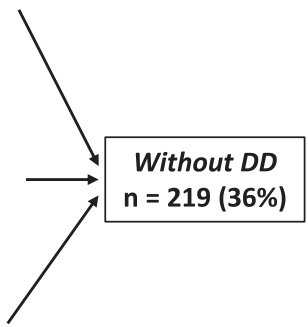
First comorbidity diagnosis $(n=67)$

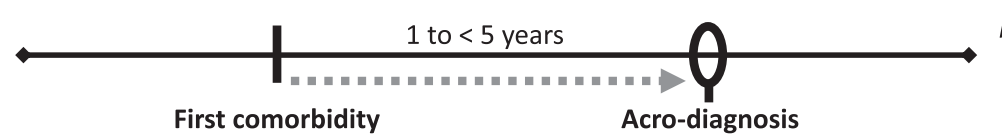

Patients with onset of comorbidity 1 year to $<5$ years before acromegaly diagnosis $(n=140)$
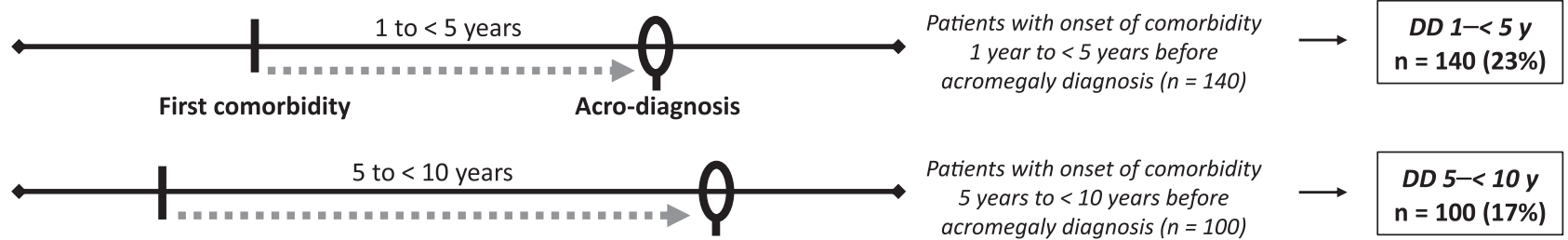

Patients with onset of comorbidity 5 years to $<10$ years before acromegaly diagnosis $(n=100)$

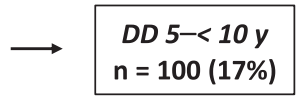
First comorbidity

Acro-diagnosis

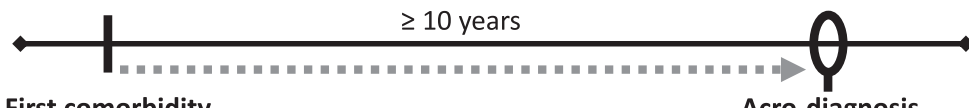

Patients with onset of comorbidity $\geq 10$ years before acromegaly

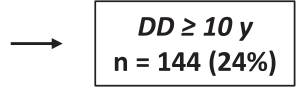

First comorbidity

Acro-diagnosis diagnosis $(n=144)$

\section{Figure 1}

Categories of diagnostic delay in patients diagnosed with acromegaly between 2001 and 2013 in Sweden. Acro, acromegaly; DD, diagnostic delay. 
following medical treatments were included: somatostatin analogues, dopamine agonists, and GH receptor antagonists. The date of any fatality was retrieved from the Cause of Death Registry in order to study the impact of the diagnostic delay on mortality.

\section{Statistical analysis}

Continuous variables were presented either as mean (s.D.) or median (minimum, maximum (min, max)) and categorical variables as number (\%). For comparison between gender, Fisher's exact test was used for dichotomous variables and Fisher's non-parametric permutation test was used for continuous variables. For comparison between ordered groups of diagnostic delay the Mantel-Haenszel chi-squared test was used for dichotomous variables and the Jonckheere-Terpstra test for continuous variables.

Person-years at risk were calculated from study inclusion (e.g. date of acromegaly diagnosis) to death or end of study, and stratified according to gender, 5-year age groups, and 1-year calendar periods. The expected number of cases for each stratum was calculated using the general Swedish population for every calendar year and 5-year age group as reference. The observed number of deaths among patients with acromegaly was compared to the expected number in the background population and standardized mortality ratios (SMRs) were calculated. Ninety-five percent confidence intervals (CIs) were calculated assuming a Poisson distribution of the observed numbers. All statistical analyses were carried out using SAS, version 9.4 .

\section{Results}

\section{Patient characteristics}

The analysis included data from 603 patients (280 men and 323 women) diagnosed with acromegaly between 2001 and 2013. The mean (s.D.) age at diagnosis was 51.8 (15.3) years and mean follow-up time was 12.2 (6.6) years (Table 1$)$. The median (min, max) patient-years at risk was $27.0(14.2,27.0)$ years. Of the 603 patients, 579 (96.0\%) had at least one comorbidity during the study period. Patients presented with a mean (s.D.) of 1.9 (1.8) comorbidities at diagnosis. Each patient had a mean of 4.1 (2.5) comorbidities during the entire study period with a higher number of comorbidities in women than men (4.3 (2.5) vs 3.7 (2.4); $P=0.003$ ) (Table 1 ). Overall, a total of 418 patients $(69.3 \%)$ had been treated with pituitary surgery and $74(12.3 \%)$ with radiotherapy. Hypopituitarism was recorded in 193 patients (32.0\%) (Table 1).

\section{Delay to diagnosis of acromegaly}

The mean (s.D.) time from the first registered comorbidity to diagnosis was 5.5 (6.2) years (median (min, max) 3.3 $(0.0,25.9)$ years). Mean diagnostic delay was $6.0(6.3)$ years in women and $5.0(6.1)$ years in men $(P=0.055$; Table 1$)$. The sub-analysis of diagnostic delay using a stricter selection of more specific comorbidities (Supplementary Table 2) led to a similar result, with a mean diagnostic delay of 4.9 (6.3) years.

In 128 patients, comorbidities were recorded either at the same time or after acromegaly was diagnosed. These patients were grouped together with patients who had no comorbidity $(n=24)$ and with those who had comorbidities occurring $<1$ year before the diagnosis of acromegaly $(n=67)$ in the group Without Diagnostic Delay ( $n=219$ patients) (Fig. 1$)$.

Overall, 140 (23.2\%) patients received the diagnosis of acromegaly 1 to $<5$ years after the onset of comorbidities (group Diagnostic Delay 1-<5). In 100 (16.6\%) patients, the delay to diagnosis of acromegaly was 5 to $<10$ years (group Diagnostic Delay 5-<10), and in 144 (23.9\%) patients the delay was $\geq 10$ years (group Diagnostic Delay 10) (Fig. 1).

The mean age at the first registered comorbidity was 46.8 (15.1) and this was significantly lower in patients with longer diagnostic delay $(P=0.0025)$. On the other hand, the mean age at diagnosis of acromegaly was higher in patients with a longer diagnostic delay $(P<0.0001)$ (Table 2$)$.

The number of comorbidities increased with longer diagnostic delay $(P<0.0001)$ (Fig. $2 \mathrm{~A}$ and Table 2$)$. When the study cohort was stratified by age at diagnosis $(<45$; $45-<60$; $\geq 60$ years), the number of comorbidities was higher in patients with longer diagnostic delay in all three age-groups (for each age-group: $P<0.0001$ ) (Table 3 ).

Overall, the most frequent comorbidities were neoplasms (pituitary neoplasms excluded) (61.0\%), and cardiovascular (56.7\%) and musculoskeletal (51.2\%) diseases (Table 2). The frequencies of neoplasms (67.2 vs $53.9 \%, \quad P=0.0012)$, endocrine-metabolic complications (50.2 vs $22.5 \% ; P=<0.0001$ ), and neuropsychiatric diseases (19.8 vs $10.0 \% ; P=0.0011$ ) were higher in women than in men. Conversely, the frequency of respiratory diseases was higher in men than women $(27.5$ vs $15.8 \% ; P<0.001)$.

When mean (s.D.) diagnostic delay was examined by comorbidity categories, the longest mean (s.D.) diagnostic 
Table 2 Characteristics of patients with acromegaly diagnosed between 2001 and 2013 by diagnostic delay.

\begin{tabular}{|c|c|c|}
\hline Characteristics & Total $(n=603)$ & $\begin{array}{l}\text { Without delay } \\
\quad(n=219)\end{array}$ \\
\hline \multicolumn{3}{|l|}{ Gender, $n(\%)$} \\
\hline Male & $280(46.4)$ & $108(49.3)$ \\
\hline Female & $323(53.6)$ & $111(50.7)$ \\
\hline Age at diagnosis, mean (s.D.) & $51.8(15.3)$ & $47.3(14.7)$ \\
\hline $\begin{array}{l}\text { Age at first comorbidity, } \\
\text { mean (s.D.) }\end{array}$ & $46.8(15.1)$ & $48.8(15.1)$ \\
\hline $\begin{array}{l}\text { Comorbidities up to } \\
\text { diagnosis, mean (S.D.) }\end{array}$ & $1.9(1.8)$ & $0.4(0.7)$ \\
\hline $\begin{array}{l}\text { Comorbidities at any } \\
\text { time, mean (s.D.) }\end{array}$ & $4.1(2.5)$ & $2.8(2.0)$ \\
\hline \multicolumn{3}{|c|}{ Frequency of comorbidities any time, $n(\%)$} \\
\hline Local effects & 277 (45.9) & $82(37.4)$ \\
\hline Hypopituitarism & $193(32.0)$ & $62(28.3)$ \\
\hline Musculoskeletal & 309 (51.2) & $70(32.0)$ \\
\hline Cardiovascular & $342(56.7)$ & $96(43.8)$ \\
\hline Respiratory & $128(21.2)$ & $27(12.3)$ \\
\hline Endocrine metabolic & $225(37.3)$ & $64(29.2)$ \\
\hline Neoplasms & $368(61.0)$ & $107(48.9)$ \\
\hline Neuropsychiatric & $92(15.3)$ & $11(5.0)$ \\
\hline Others & $89(14.8)$ & $30(13.7)$ \\
\hline
\end{tabular}

\begin{tabular}{|c|c|c|c|}
\hline $\begin{array}{c}\text { Delay } \mathbf{1}-<\mathbf{5} \mathbf{y} \\
(n=140)\end{array}$ & $\begin{array}{c}\text { Delay } 5-<\mathbf{1 0} y \\
(n=100)\end{array}$ & $\begin{array}{c}\text { Delay } \geq 10 \mathbf{y} \\
(n=144)\end{array}$ & $P$ value \\
\hline $70(50.0)$ & $45(45.0)$ & 57 (39.6) & \\
\hline $70(50.0)$ & 55 (55.0) & $87(60.4)$ & 0.059 \\
\hline $51.1(14.9)$ & $52.2(14.1)$ & $59.2(14.4)$ & $<0.0001$ \\
\hline $48.1(14.9)$ & $45.1(14.0)$ & 44.1 (15.5) & 0.0025 \\
\hline $2.1(1.2)$ & $2.8(1.5)$ & $3.4(1.8)$ & $<0.0001$ \\
\hline $4.1(2.3)$ & $4.9(2.3)$ & $5.4(2.4)$ & $<0.0001$ \\
\hline $71(50.7)$ & $57(57.0)$ & $67(46.5)$ & 0.031 \\
\hline $50(35.7)$ & $35(35.0)$ & 46 (31.9) & 0.42 \\
\hline 75 (53.6) & $62(62.0)$ & $102(70.8)$ & $<0.0001$ \\
\hline $77(55.0)$ & 63 (63.0) & 106 (73.6) & $<0.0001$ \\
\hline $37(26.4)$ & $31(31.0)$ & 33 (22.9) & 0.0042 \\
\hline 55 (39.3) & $36(36.0)$ & 70 (48.6) & 0.0005 \\
\hline $85(60.7)$ & 71 (71.0) & 105 (72.9) & $<0.0001$ \\
\hline 18 (12.9) & $24(24.0)$ & 39 (27.1) & $<0.0001$ \\
\hline 19 (13.6) & 14 (14.0) & $26(18.1)$ & 0.28 \\
\hline
\end{tabular}

delay was found for neuropsychiatric diseases at 4.6 (6.3) years and the shortest for local effects of the pituitary tumour at 1.7 (3.4) years (Fig. 3). Mean time from diagnosis of neoplasms, and musculoskeletal and cardiovascular diseases to diagnosis of acromegaly was 3.7 (5.4), 3.4 (4.9), and 2.6 (4.5) years, respectively. Mean diagnostic delay for neoplasms was longer in women than in men (4.5 (6.0) vs 2.7 (4.3) years; $P=0.0018$ ), whereas no gender differences in diagnostic delay were found for the other comorbidity categories (Fig. 3).

\section{Treatment patterns and diagnostic delay}

The frequency of pituitary surgery was lower in patients with longer diagnostic delay, with $73.1 \%$ of patients treated with surgery in the group without diagnostic delay and $61.1 \%$ in the group with diagnostic delay $\geq 10$ years $(P=0.017)$ (Table 4). Overall, $74(12.3 \%)$ patients were treated with radiotherapy and the frequency of radiotherapy was similar across the diagnostic delay groups ( $(P=0.98)$ (Table 4$)$. The proportion of patients who had been treated with radiotherapy alone increased with longer diagnostic delay $(P=0.005)$. For all 603 patients, neither pituitary surgery nor radiotherapy was recorded in $174(28.9 \%)$ with no differences among the different diagnostic delay groups (Table 4).

Data on pharmacotherapy were available for a subgroup of 358 patients ( 170 men, 188 women) who had been diagnosed after 1 July 2005. In this subgroup, 271
(75.7\%) patients had been treated with pituitary surgery, 44 (12.3\%) with radiotherapy, and 196 (54.7\%) with pharmacotherapy at any time during the study period. In this subgroup of patients, no differences in treatment pattern were recorded across the different diagnostic delay groups.

\section{Mortality by diagnostic delay}

In total, the observed number of deaths was 61 compared to the expected number of 42.2, resulting in an SMR of 1.45 (95\% CI: 1.11 to 1.86 ). When analysing the effect of different length of diagnostic delay on mortality, a statistically significant increase in mortality was only found in the group of patients with the longest diagnostic delay (Diagnostic Delay $\geq 10$ years) (SMR 1.76, 95\% CI: 1.12 to 2.65$)(P=0.016)$. Mortality in the other groups was not significantly different compared to the general population. The numerically lowest SMR was observed in the group without diagnostic delay $(1.18,95 \%$ CI: 0.68 to 1.92) (Fig. 2B).

\section{Discussion}

This is the first attempt to systematically analyse diagnostic delay and its impact on morbidity and mortality, in a nationwide, unselected cohort of patients with acromegaly. We recorded a mean delay of 5.5 years, 

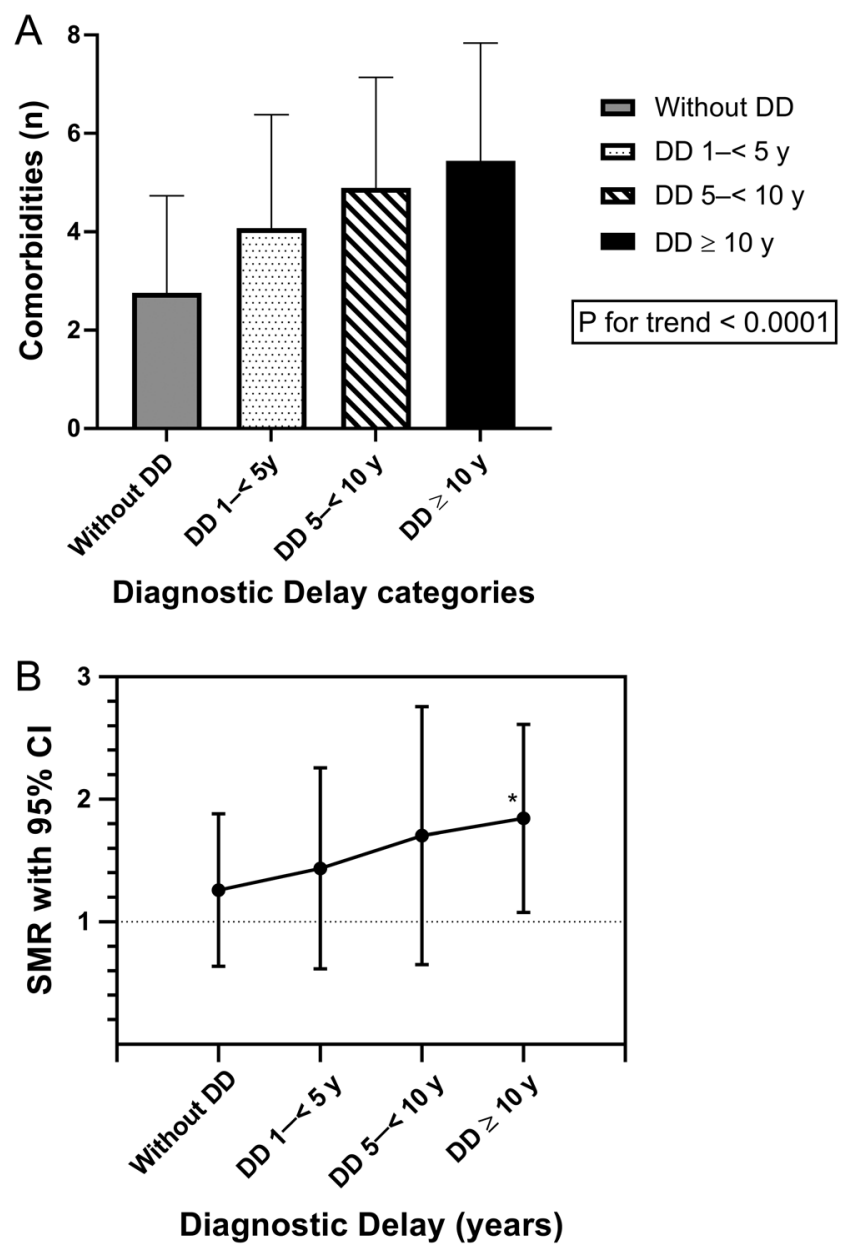

Figure 2

(A) Mean (S.D.) number of comorbidities registered in patients with acromegaly in Sweden throughout the entire study period increased significantly $(P<0.0001)$ with longer diagnostic delay. (B) Mortality in patients with acromegaly in Sweden by diagnostic delay. Statistically significant increase in mortality was only recorded in patients with a diagnostic delay $\geq 10$ years, with an SMR of 1.76 (95\% Cl: 1.12 to 2.65$)$. In the other groups mortality was not significantly different compared to the background population. ${ }^{*} P<0.016$. DD, diagnostic delay; SMR, standardized mortality ratio.

with a large number of patients receiving the diagnosis more than 10 years after the first acromegaly associated comorbidity. Overall, $96 \%$ of patients presented at least one complication during the study period and patients with longer diagnostic delay had a higher number of complications. Excess mortality was only found in patients with the longest diagnostic delay.

Delay in diagnosis of acromegaly has previously been described in only a few trials but none of them was specifically designed to analyse this issue. Recent multicentre studies have shown a delay ranging between 5 and 14 years, which has not changed over the last decades $(13,16,17)$. However, the methods used to estimate the diagnostic delay (e.g. interviews, or questionnaires) have been imprecise and susceptible to bias. The current study is the first study analysing diagnostic delay based on objective data from national health registries.

The impact of diagnostic delay on morbidity in patients with acromegaly is unknown. Our study is the first to investigate this, describing an increasing number of comorbidities with longer diagnostic delay both at diagnosis and at any time during follow-up. During the entire study period, the group without diagnostic delay had a mean total of 2.8 comorbidities increasing to 5.4 in patients with a diagnostic delay of $\geq 10$ years. One could argue that higher age at diagnosis in patients with a longer diagnostic delay could affect our results. However, it is important to note that the mean age at the first comorbidity was significantly lower in patients with longer diagnostic delay (Table 2). This suggests that higher age at diagnosis was an effect of the delay in the diagnostic process. Therefore, the analysis was not adjusted for age at diagnosis. Consistent with this, when the study cohort was stratified by age at diagnosis $(<45$; $45-<60 ; \geq 60$ years), similar findings were observed, with a higher number of comorbidities in patients with longer diagnostic delay in all three age-groups (Table 3). This subanalysis shows that age is not the main determinant of morbidity in acromegaly, rather the delay in the diagnostic process plays a crucial role.

Comorbidities were recorded in the majority of patients and only 24 patients, in the group without diagnostic delay, were free of complications during the entire study period. The most frequent comorbidities were neoplasms (excluding pituitary neoplasms) and cardiovascular and musculoskeletal diseases. In previous registry studies, hypertension and type 2 diabetes have been described as the most common complications $(12,16)$. However, frequency of comorbidities varies greatly across registries due to the method used for data collection; for example, uncomplicated hypertension may not be recorded in our national patient registry, whereas in acromegaly registries this comorbidity will be specifically captured.

In our study, a strong tendency toward a longer diagnostic delay was found in women compared to men. In addition, the mean number of comorbidities at diagnosis as well as during the entire study period was higher in women than men. In contrast to our study, the German Registry reported a longer diagnostic delay in men than 
Table 3 Number of acromegaly associated comorbidities in diagnostic delay subgroups stratified by age. Data re presented as mean (S.D.)

\begin{tabular}{l}
\hline Number of comorbidities \\
\hline Comorbidities up to diagnosis \\
Entire cohort \\
Patients $<45$ years at diagnosis \\
Patients $45-<60$ years at diagnosis \\
Patients $\geq 60$ years at diagnosis \\
Comorbidities at any time \\
Entire cohort \\
Patients $<45$ years at diagnosis \\
Patients $45-<60$ years at diagnosis \\
Patients $\geq 60$ years at diagnosis
\end{tabular}

\begin{tabular}{ccc}
\hline \multicolumn{1}{c}{$\boldsymbol{n}$} & & Total \\
\cline { 1 - 1 }$(n=603)$ & & $1.9(1.8)$ \\
$(n=207)$ & & $1.4(1.4)$ \\
$(n=208)$ & & $1.8(1.7)$ \\
$(n=188)$ & & $2.6(2.0)$ \\
& & \\
$(n=603)$ & & $4.1(2.5)$ \\
$(n=207)$ & & $3.0(2.1)$ \\
$(n=208)$ & & $4.2(2.4)$ \\
$(n=188)$ & & $5.1(2.4)$ \\
&
\end{tabular}

\begin{tabular}{c}
$\begin{array}{c}\text { Without } \\
\text { delay }\end{array}$ \\
\hline \\
$0.4(0.7)$ \\
$0.4(0.6)$ \\
$0.4(0.7)$ \\
$0.4(0.7)$ \\
\\
$2.8(2.0)$ \\
$2.0(1.7)$ \\
$3.1(2.1)$ \\
$3.8(1.8)$ \\
\hline
\end{tabular}

\begin{tabular}{c}
$\begin{array}{c}\text { Delay } \\
\mathbf{1 -}<\mathbf{5} \mathbf{y}\end{array}$ \\
\hline $2.1(1.2)$ \\
$1.9(1.1)$ \\
$2.1(1.1)$ \\
$2.4(1.5)$ \\
$4.1(2.3)$ \\
$3.3(2.0)$ \\
$4.4(2.4)$ \\
$4.6(2.4)$ \\
\hline
\end{tabular}

\begin{tabular}{c}
$\begin{array}{c}\text { Delay } \\
\mathbf{5 - < 1 0} \mathbf{y}\end{array}$ \\
\hline $2.8(1.5)$ \\
$2.3(1.1)$ \\
$2.8(1.5)$ \\
$3.3(2.0)$ \\
$4.9(2.3)$ \\
$4.0(2.2)$ \\
$5.1(2.0)$ \\
$5.6(2.4)$ \\
\hline
\end{tabular}

\begin{tabular}{c}
\hline $\begin{array}{c}\text { Delay } \\
\mathbf{1 0} \mathbf{y}\end{array}$ \\
\hline \\
$3.4(1.8)$ \\
$2.6(2.1)$ \\
$3.2(1.7)$ \\
$3.8(1.7)$ \\
\\
$5.4(2.4)$ \\
$4.4(2.0)$ \\
$5.3(2.5)$ \\
$5.9(2.4)$ \\
\hline
\end{tabular}

in women (6 vs 5 years; $P<0.001$ ) (13). Interestingly, in a recent review including data from 19 national acromegaly registries involving more than 16,000 patients (16), it was shown that the age at diagnosis was significantly higher in women than in men. Whether this difference is due to a longer diagnostic delay in women is still unclear. A longer diagnostic delay and a higher number of comorbidities in women may follow the same pattern that has been seen for other diseases such as coronary heart disease and diabetes mellitus $(18,19)$, suggesting unequal healthcare or greater difficulties in diagnosing some diseases in women than in men.

We found that neurological and psychiatric disorders, including mental disorders and depression, may be early presenting symptoms in acromegaly, which were reported on average 4.6 years prior to diagnosis. It has previously been shown that acromegaly is associated with neuropsychiatric

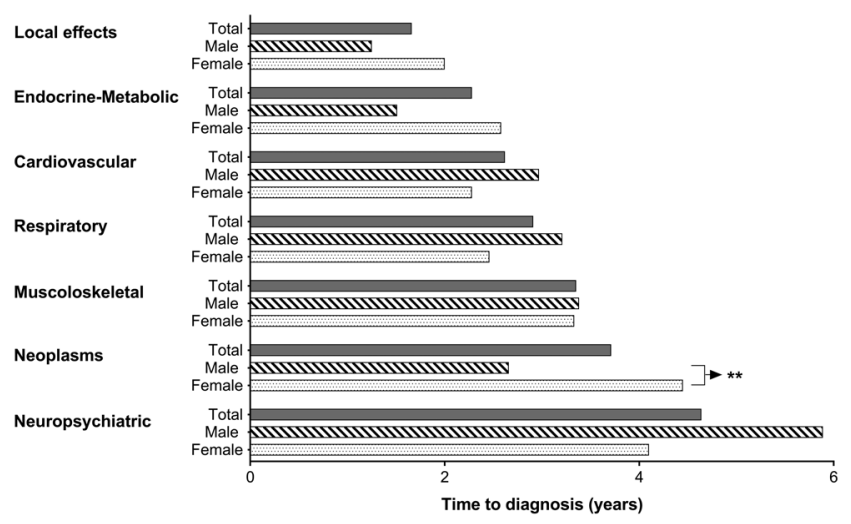

\section{Figure 3}

Mean diagnostic delay by category of comorbidity. The longest diagnostic delay was found for neuropsychiatric diseases and the shortest for local effects of the pituitary tumour. The diagnostic delay for neoplasms was longer in women than in men (4.5 vs 2.7 years). ${ }^{*} P=0.0018$.

complications such as depressive symptoms and anxiety, leading to impaired quality of life $(20,21,22,23)$. There is some evidence showing that, despite long-term biochemical remission, patients have persistent decreased quality of life (24). Interestingly, in a prospective study including 80 patients (25), it has been reported that quality of life in acromegaly is independent of biochemical control. On the other hand, data from Germany showed that there is a significant correlation between diagnostic delay and quality of life, psychological well-being, and depression (26). In agreement with this, we found that the proportion of patients with neurological and psychiatric disorders increased with longer diagnostic delay, for example, 5\% of patients in the group diagnosed without delay compared to $27 \%$ in the group with the longest diagnostic delay.

The mean diagnostic delay for neoplasms was 3.7 years, which was longer in women than in men (Fig. 3). Musculoskeletal and cardiovascular diseases occurred on average 3.4 and 2.6 years, respectively, prior to diagnosis. These are important findings since they may raise medical awareness of signs and symptoms of acromegaly and possibly reduce the delay in diagnosis.

There is considerable evidence that mortality in acromegaly has improved over time from being 2-3 fold increased to being almost the same as the general population $(6,27)$. It is well known that biochemical control plays an important role for long-term outcome (7, 28). On the other hand, there are no data on the effect of diagnostic delay on mortality. Our results suggest that diagnostic delay has an impact on mortality, with excess mortality only in patients with a diagnostic delay $\geq 10$ years and the numerically lowest SMR in patients without diagnostic delay.

In this study, a total of $69 \%$ of patients were treated with pituitary surgery, which is in agreement with other recent registry studies $(11,12)$. The use of pituitary 
Table 4 Treatment patterns by diagnostic delay in patients with acromegaly in Sweden diagnosed between 2001 and 2013 . Data are presented as $n(\%)$.

\begin{tabular}{|c|c|c|}
\hline Treatment & Total $(n=603)$ & $\begin{array}{c}\text { Without delay } \\
\quad(n=219)\end{array}$ \\
\hline Any surgery & $418(69.3)$ & $160(73.1)$ \\
\hline Any radiotherapy & $74(12.3)$ & $29(13.2)$ \\
\hline Only surgery & $355(58.9)$ & $132(60.3)$ \\
\hline Only radiotherapy & $11(1.8)$ & $1(0.5)$ \\
\hline Surgery and radiotherapy & $63(10.4)$ & $28(12.8)$ \\
\hline Neither surgery nor radiotherapy & $174(28.9)$ & $58(26.5)$ \\
\hline
\end{tabular}

\begin{tabular}{|c|}
\hline $\begin{array}{c}\text { Delay } \mathbf{1}-<\mathbf{5} \mathbf{y} \\
(n=140)\end{array}$ \\
\hline 101 (72.1) \\
\hline 15 (10.7) \\
\hline 88 (62.9) \\
\hline $2(1.4)$ \\
\hline $13(9.3)$ \\
\hline $37(26.4)$ \\
\hline
\end{tabular}

\begin{tabular}{c}
$\begin{array}{c}\text { Delay } \mathbf{5 - < 1 0 y} \\
(n=100)\end{array}$ \\
\hline $69(69.0)$ \\
$10(10.0)$ \\
$60(60.0)$ \\
$1(1.0)$ \\
$9(9.0)$ \\
$30(30.0)$ \\
\hline
\end{tabular}

\begin{tabular}{cll}
$\begin{array}{c}\text { Delay } \geq \mathbf{1 0} \mathbf{y} \\
(n=144)\end{array}$ & & P value \\
\cline { 1 - 1 } \cline { 1 - 1 } $88(61.1)$ & & 0.017 \\
$20(13.9)$ & & 0.98 \\
$75(52.1)$ & & 0.13 \\
$7(4.9)$ & & 0.0051 \\
$13(9.0)$ & & 0.23 \\
$49(34.0)$ & & 0.11 \\
\hline
\end{tabular}

surgery progressively decreased in patients with longer diagnostic delay, whereas the use of radiotherapy and pharmacotherapy was similar among the groups. The reduced use of surgery with increasing diagnostic delay is likely due to the fact that patients receiving the diagnosis with longer delay are older and have a higher number of complications and, thus, higher risk of surgical complications.

The major strengths of our study are the large nationwide population, the long follow-up period, and the reliability of data, which was retrieved from official national health registries. In order to secure the correct diagnosis of patients to be included into the analysis, we used very conservative search criteria, that is, patients included needed to have both a diagnosis of acromegaly and a pituitary adenoma. In addition, the study design is another important strength of this study, which allowed analysing the diagnostic delay based on objective and accurate data from national health registries. Moreover, all patients included in the study had the same time at risk to develop complications before the diagnosis of acromegaly. However, there are some limitations to be considered. Data on clinical characteristics, biochemical activity, and pituitary tumour size were not available in the registries. Using the first complication as starting date to calculate the diagnostic delay may be considered as a limitation because the diagnostic delay may have been underestimated. However, there is no reliable method to objectively record the first symptom/sign related to acromegaly, whereas data on the first registered comorbidity are objective and accurate. Indeed, the method used in this study is more reliable than those used in previous studies based on medical interviews and questionnaires, where an inaccurate recall of signs and symptoms of acromegaly could have biased the results. One could argue that some of the predefined complications used in this study are rather unspecific in acromegaly. To overcome this problem a sub-analysis of diagnostic delay based on a smaller group of more specific complications
(Supplementary Table 2) has been performed, showing a similar delay to diagnosis.

In conclusion, in the majority of patients with acromegaly, the diagnosis is delayed for several years, with a large number of patients receiving the diagnosis more than 10 years after onset of the first comorbidity. Prolonged diagnostic delay is associated with increased numbers of comorbidities and excess mortality. Efforts should be made to increase awareness of acromegaly among clinicians so that it can be recognized and treated earlier to improve long-term outcome.

\section{Supplementary materials}

This is linked to the online version of the paper at https://doi.org/10.1530/ EJE-20-0019.

\section{Declaration of interest}

$D$ E has received lecture fees from Ipsen. O R has received lecture fees from Novo Nordisk, Ipsen, Sandoz, and Pfizer, an unrestricted research grant from HRA-pharma, and consultancy fees from Novartis and HRApharma. G J has served as a consultant for Novo Nordisk, Shire, and Astra Zeneca, and has received lecture fees from Eli Lilly, Ipsen, Novartis, Novo Nordisk, Merck Serono, Otsuka, and Pfizer. G J is serving as an Editor for the European Journal of Endocrinology. D S O has been a consultant for Sandoz, Ipsen, and Pfizer. Gudmundur Johannsson is on the editorial board of EJE. Gudmundur Johannsson was not involved in the review or editorial process for this paper, on which he/she is listed as an author.

\section{Funding}

This study was supported by the Swedish government under the ALF agreement (ALFGBG-719531).

\section{Acknowledgments}

The authors are indebted to The National Board of Health and Welfare for their excellent collaboration. The authors are grateful to the statisticians Anders Pehrsson and Mattias Molin of the Statistiska Konsultgruppen (Gothenburg, Sweden) for performing all the statistical analyses in the study. The authors acknowledge Daniel Granfeldt, Pharmalex (Gothenburg, Sweden) for coordination of the project. They also would like to thank Peter Todd (Tajut Ltd., Kaiapoi, New Zealand) for third-party writing assistance in drafting of this manuscript, for which he received financial compensation from ALF funding. 


\section{References}

1 Melmed S. Medical progress: acromegaly. New England Journal of Medicine 2006355 2558-2573. (https://doi.org/10.1056/ NEJMra062453)

2 Colao A, Ferone D, Marzullo P \& Lombardi G. Systemic complications of acromegaly: epidemiology, pathogenesis, and management. Endocrine Reviews 200425 102-152. (https://doi. org/10.1210/er.2002-0022)

3 Lesén E, Granfeldt D, Houchard A, Dinet J, Berthon A, Olsson DS, Björholt I \& Johannsson G. Comorbidities, treatment patterns and cost-of-illness of acromegaly in Sweden: a register-linkage population-based study. European Journal of Endocrinology 2017176 203-212. (https://doi.org/10.1530/EJE-16-0623)

4 Rajasoorya C, Holdaway IM, Wrightson P, Scott DJ \& Ibbertson HK. Determinants of clinical outcome and survival in acromegaly. Clinical Endocrinology 199441 95-102. (https://doi. org/10.1111/j.1365-2265.1994.tb03789.x)

5 Sherlock M, Ayuk J, Tomlinson JW, Toogood AA, Aragon-Alonso A, Sheppard MC, Bates AS \& Stewart PM. Mortality in patients with pituitary disease. Endocrine Reviews 201031 301-342. (https://doi. org/10.1210/er.2009-0033)

6 Decreasing mortality and changes in treatment patterns in patients with acromegaly from a nationwide study. European Journal of Endocrinology 2019178 X1-X3. (https://doi.org/10.1530/EJE-18-0015e)

7 Holdaway IM, Rajasoorya RC \& Gamble GD. Factors influencing mortality in acromegaly. Journal of Clinical Endocrinology and Metabolism 200489 667-674. (https://doi.org/10.1210/jc.2003031199)

8 Gordon DA, Hill FM \& Ezrin C. Acromegaly: a review of 100 cases. Canadian Medical Association Journal 196287 1106-1109.

9 Nabarro JD. Acromegaly. Clinical Endocrinology 198726 481-512. (https://doi.org/10.1111/j.1365-2265.1987.tb00805.x)

10 Reid TJ, Post KD, Bruce JN, Nabi Kanibir M, Reyes-Vidal CM \& Freda PU. Features at diagnosis of 324 patients with acromegaly did not change from 1981 to 2006: acromegaly remains underrecognized and under-diagnosed. Clinical Endocrinology 201072 203-208. (https://doi.org/10.1111/j.1365-2265.2009.03626.x)

11 Arosio M, Reimondo G, Malchiodi E, Berchialla P, Borraccino A, De Marinis L, Pivonello R, Grottoli S, Losa M, Cannavò S et al. Predictors of morbidity and mortality in acromegaly: an Italian survey. European Journal of Endocrinology 2012167 189-198. (https://doi.org/10.1530/ EJE-12-0084)

12 Maione L, Brue T, Beckers A, Delemer B, Petrossians P, Borson-Chazot F, Chabre $\mathrm{O}$, François $\mathrm{P}$, Bertherat J, Cortet-Rudelli $\mathrm{C}$ et al. Changes in the management and comorbidities of acromegaly over three decades: the French Acromegaly Registry. European Journal of Endocrinology 2017176 645-655. (https://doi.org/10.1530/EJE-16-1064)

13 Petersenn S, Buchfelder M, Gerbert B, Franz H, Quabbe HJ, Schulte HM, Grussendorf M, Reincke M \& Participants of the German Acromegaly Register. Age and sex as predictors of biochemical activity in acromegaly: analysis of 1485 patients from the German Acromegaly Register. Clinical Endocrinology 200971 400-405. (https://doi.org/10.1111/j.1365-2265.2009.03547.x)

14 Ludvigsson JF, Andersson E, Ekbom A, Feychting M, Kim JL, Reuterwall C, Heurgren M \& Olausson PO. External review and validation of the Swedish national inpatient register. BMC Public Health 201111 450. (https://doi.org/10.1186/1471-2458-11-450)

15 Johansson LA. Dödsorsaksstatistik-Historik, produktionsmetoder och tillförlitlighet. The National Board of Health and Welfare, 2010. (available at: https://www.socialstyrelsen.se/globalassets/ sharepoint-dokument/artikelkatalog/statistik/2010-4-33.pdf). Accessed on 13 Sep 2019.

16 Maione L \& Chanson P. National acromegaly registries. Best Practice and Research: Clinical Endocrinology and Metabolism 201933101264. (https://doi.org/10.1016/j.beem.2019.02.001)

17 Dal J, Feldt-Rasmussen U, Andersen M, Kristensen LØ, Laurberg P, Pedersen L, Dekkers OM, Sørensen HT \& Jørgensen JO. Acromegaly incidence, prevalence, complications and long-term prognosis: a nationwide cohort study. European Journal of Endocrinology 2016175 181-190. (https://doi.org/10.1530/EJE-16-0117)

18 Shah T, Palaskas N \& Ahmed A. An update on gender disparities in coronary heart disease care. Current Atherosclerosis Reports 20161828. (https://doi.org/10.1007/s11883-016-0574-5)

19 Arnetz L, Ekberg NR \& Alvarsson M. Sex differences in type 2 diabetes: focus on disease course and outcomes. Diabetes, Metabolic Syndrome and Obesity: Targets and Therapy 20147 409-420. (https:// doi.org/10.2147/DMSO.S51301)

20 Anagnostis P, Efstathiadou ZA, Charizopoulou M, Selalmatzidou D, Karathanasi E, Poulasouchidou M \& Kita M. Psychological profile and quality of life in patients with acromegaly in Greece. Is there any difference with other chronic diseases? Endocrine 201447 564-571. (https://doi.org/10.1007/s12020-014-0166-5)

21 Sievers C, Dimopoulou C, Pfister H, Lieb R, Steffin B, Roemmler J, Schopohl J, Mueller M, Schneider HJ, Ising M et al. Prevalence of mental disorders in acromegaly: a cross-sectional study in 81 acromegalic patients. Clinical Endocrinology 2009 71 691-701. (https://doi.org/10.1111/j.1365-2265.2009.03555.x)

22 Sievers C, Ising M, Pfister H, Dimopoulou C, Schneider HJ, Roemmler J, Schopohl J \& Stalla GK. Personality in patients with pituitary adenomas is characterized by increased anxiety-related traits: comparison of 70 acromegalic patients with patients with non-functioning pituitary adenomas and age- and gender-matched controls. European Journal of Endocrinology 2009160 367-373. (https://doi.org/10.1530/EJE-08-0896)

23 Rapaport MH, Clary C, Fayyad R \& Endicott J. Quality-of-life impairment in depressive and anxiety disorders. American Journal of Psychiatry 2005162 1171-1178. (https://doi.org/10.1176/appi. ajp.162.6.1171)

24 Biermasz NR, van Thiel SW, Pereira AM, Hoftijzer HC, van Hemert AM, Smit JW, Romijn JA \& Roelfsema F. Decreased quality of life in patients with acromegaly despite long-term cure of growth hormone excess. Journal of Clinical Endocrinology and Metabolism 2004 89 5369-5376. (https://doi.org/10.1210/jc.2004-0669)

25 Geraedts VJ, Dimopoulou C, Auer M, Schopohl J, Stalla GK \& Sievers C. Health outcomes in acromegaly: depression and anxiety are promising targets for improving reduced quality of life. Frontiers in Endocrinology 20145 229. (https://doi.org/10.3389/fendo.2014.00229)

26 Siegel S, Streetz-van der Werf C, Schott JS, Nolte K, Karges W \& Kreitschmann-Andermahr I. Diagnostic delay is associated with psychosocial impairment in acromegaly. Pituitary 201316 507-514. (https://doi.org/10.1007/s11102-012-0447-z)

27 Dekkers OM, Biermasz NR, Pereira AM, Romijn JA \& Vandenbroucke JP. Mortality in acromegaly: a metaanalysis. Journal of Clinical Endocrinology and Metabolism 200893 61-67. (https://doi. org/10.1210/jc.2007-1191)

28 Biermasz NR, Dekker FW, Pereira AM, van Thiel SW, Schutte PJ, van Dulken H, Romijn JA \& Roelfsema F. Determinants of survival in treated acromegaly in a single center: predictive value of serial insulin-like growth factor I measurements. Journal of Clinical Endocrinology and Metabolism 200489 2789-2796. (https://doi. org/10.1210/jc.2003-032041)

Received 7 January 2020

Revised version received 10 March 2020

Accepted 26 March 2020 